\title{
Knowledge, Attitude and Practices Towards Tuberculosis: Study Amongst Urban Adults Visiting the Community Health Center
}

\author{
Reji Laiby ${ }^{1, *}$, Pal Debjani ${ }^{1}$, Bhandarkar Prashant ${ }^{2}$
}

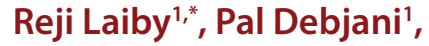
Bhandarkar Prashant ${ }^{2}$

'Mandala Dispensary, Medical Division, Bhabha Atomic Research Centre, Mumbai, Maharashtra, INDIA.

2Department of Statistics, Medical Division, Bhabha Atomic Research Centre, Mumbai, Maharashtra, INDIA.

\section{Correspondence}

Mrs. Laiby Reji

Mandala Dispensary, Medical Division, Bhabha Atomic Research Centre, Mumbai-400094, Maharashtra, INDIA. Email: laiby.reji@gmail.com

\section{History}

- Submission Date: 16-11-2021

- Revised Date: 03-12-2021

- Accepted Date: 29-12-2021

DOI : 10.5530/ijmedph.2022.1.6

\section{Article Available online}

http://www.jimedph.org/v12/i1

\section{Copyright}

(C) 2022 Phcog.Net. This is an openaccess article distributed under the terms of the Creative Commons Attribution 4.0 International license.

\begin{abstract}
Background: Tuberculosis (TB) constitutes the major challenge as a Communicable disease in the world. Its impacts are higher in resource-constrained settings. Objectives: We wish to explore the knowledge-attitude-practice (KAP) towards TB among the primary health center visitors in Mumbai. Methods: The cross-sectional-self-administered interview-based survey was conducted at a primary health center of a community healthcare setup at Mumbai. Experiences of study participants, with and without family history of TB towards KAP were compared. Results: We obtained 130 valid responses (65\% were females), $28 \%$ reported to have history of TB to self or family members. In total responders $60 \%$ of said to have good knowledge about TB, $88.5 \%$ of respondents seen to have favarouable attitude, and $73.1 \%$ informed following good preventing TB practices. Conclusion: Among total responders, two in five, eight in ten and seven in ten had a good knowledge attitude and practice about TB respectively. Among the participants with and without history of TB and we found no differences in KAP parameters.

Key words: Tuberculosis, Knowledge, Attitude, Practice, Community.
\end{abstract}

\section{INTRODUCTION}

Tuberculosis (TB) burden has consistently been associated with various societies' sections with dominance in specific geographical locations. The last few decades have witnessed global, national, and regional efforts towards the control of TB. ${ }^{1}$ Despite all the efforts globally, more than 9.5 million people get infected with $\mathrm{TB}$, among which $17 \%$ (1.7 million) die per year. ${ }^{2}$ When it comes to India, infections due to TB are the second-highest infectious cause of death in India. ${ }^{1,3}$ Revised National TB Control Programme (RNTCP) progressively addressed TB control in the country. Similarly, the National Strategic Plan (NSP) 2017-2025 to eliminate TB has emerged with innovative steps. ${ }^{4}$ Although due to massive policy efforts, there was a $10.9 \%$ decadal reduction in TB deaths in absolute numbers fatalities.

TB is caused by bacteria Mycobacterium tuberculae, which most often affect the lungs. TB is curable, and prevention can help to control the spread. When people with pulmonary TB cough, sneeze or spit, they propel the TB germs into the air. A person needs to inhale only a few of these germs to become infected. About one-third of the world's population has latent TB, which means people have been infected by TB bacteria but are not (yet) ill with the disease and cannot transmit the disease. India bears one-fourth of the global TB burden with $26 \%$ cases of fatalities due to TB.
Raising community awareness can help to control the spread of disease. Positive associations in knowledge with treatment adherence and prevention is reported in studies. While stigma is seen to be associated with avoiding or delaying treatment or poor compliance., Incorrect knowledge or awareness leads to stigmatization and discrimination in $\mathrm{TB}$ and myths about $\mathrm{TB} .^{7}$ Having appropriate or adequate knowledge helps address psycho-social factors adequately. Considering the significance of knowledge attitude and practice (KAP), we decided to explore these factors' relationship with TB. Study will also delve into comparision of KAP differences among respondent with and without the family history of the TB.

\section{METHODOLOGY}

A prospective observational interview-based study was performed. The study location was the dispensary of the comprehensive healthcare service scheme (CHSS) at Mumbai, located at Mandala. Beneficiaries of CHS scheme comprises of urban, educated and middle-income population. Details about the healthcare setup and the scheme are published elsewhere. ${ }^{8,9}$ Study participants were selected from the beneficiaries visiting the study center during the period starting from 08/2019 to $12 / 2019$. The visiting patients or their companion of the age more than 18 years of were potential
Cite this article : Laiby R, Debjani P, Prashant B. Knowledge, Attitude and Practices Towards Tuberculosis: Study Amongst Urban Adults Visiting the Community Health Center. Int J Med Public Health. 2022;12(1):28-32. 
Laiby, et al.: Knowledge, Attitude and Practices towards Tuberculosis

eligible for this study. Those who were unable to read and respond to the questionnaire were excluded from the study. Persons who expressed their willingness to participate in the survey were briefed about the research and the details. Those who agreed to participate in the study were requested for signed consent form and to respond the self-administered questionnaire. Considering baseline population of 11500 individuals mapped to the study dispensary and considering precision of $8 \%$, we calculated sample size of 130 individuals for this study.

The questionnaire was designed based on various approaches about KAP-related aspects. We obtained content and face validity based on the pilot of five responses. The self-explanatory, non-intentional nature of questions was considered in the questionnaire after the piloting. Fifteen question of background characteristics, followed by eight about knowledge, ten about attitude and six questions about practices were considered for this study. All the responses received were anonymously considered for the purpose of the inference.

The score of each correct response was calculated for knowledge and practice. For attitude, five Likert-scale-based questions excluded from the score while the remaining five were considered for the score calculation. Total correct responses were grouped across those participants who had a family history of TB or those who have ever been diagnosed with the TB. Overall, KAP characteristics were compared across socio-economic parameters of the study responders.

All the data were analyzed using Microsoft Office Excel 2019 for Windows and Statistical Package for Social Sciences (SPSS version 25) for Windows. Responders' data were analyzed for socio-demographic parameters across variables under consideration. Total responders were compared between those individuals whoever had a history of TB in their family as well as those who ever was diagnosed with the disease. The score of correct and non-correct responses were given for the KAP domain for each socio-demographic parameter. Cross-tabulation data with row percentages were shown for analysis. Chi-square test of significance and non-parametric test applied. $P$-value below 0.05 was considered to be statistically significant.

\section{RESULTS}

Overall, 130 participants with valid records were considered for the study. Out of the total respondents, $65 \%$ were females, and $35 \%$ were males. The majority of participants (43\%) are from the $31-40$ years age group. $80 \%$ of the study population were living in a nuclear family group. (Table 1) There were $13 \%$ of respondents reported to be diagnosed with TB in the past. While $21 \%$ reported to have family member ever diagnosed with TB. Among respondernt demogrphic characteristics and their (or family member) history of TB diagnosed no statistically significant difference was found except family types $(p>0.05)$. In nuclear family 32.4 reported to have family history of TB same was $8 \%$ in joint family repsonders ( $p$ value 0.014 ). Three in five respoders reported to have good and adequate knowledge about the TB. Nine in ten responder seem to have positive or favourable attitude towards the practices to avoid TB occurance or spread. Three in four reported to have agreed for the good practices.

History of TB to the responder or in the family seems to have no effect in TB related KAP responses of an individual. No statistically significant difference was noticed in TB related responses ( $p$ value $>0.05$ ).

\section{Knowledge about TB.}

From overall respondents, $60 \%$ of participants had good knowledge about TB (knowledge score value is more significant than mean knowledge score value). $58.46 \%$ of participants reported that TB occurs due to bacteria. $14.62 \%$ reported that it occurs genetically. Only $33.08 \%$ of participants mentioned that they know about the BCG vaccine for TB. $53.85 \%$ of participants were fully aware of TB symptoms. Only $6.92 \%$ of respondents know about all the modes of the transmission of TB. The majority of respondents $(83.08 \%)$ think that TB is curable and the treatment for the same is free of cost $(80 \%)$.

\section{Attitude towards TB}

Overall, favorable attitude present in the community towards TB (88.05\%). The majority of respondents $(86.15 \%)$ are willing to share their information about TB with others. Also, people favor undergoing family screening to detect Tuberculosis if any family member is diagnosed with TB $(85.38 \%)$. The majority of participants $(88.46 \%)$ also believe that wearing a mask in pulmonary $\mathrm{TB}$ is a preventable measure to stop the spreading of TB. Only $27.69 \%$ of respondents believe that interaction with TB Patients Contributes to possible stigma.

\section{Prevention practice for TB}

Most of the respondents have done immunization for their child with BCG (82.31\%). Almost all (98.46\%) the participants maintain the proper hygiene at home. Only one-seventh of participants attended a health education program for prevention and management of TB. Seven in eight participants have an adequately ventilated house. $76.92 \%$ of participants mentioned that nutrition plays an essential role in tuberculosis management. (Table 2).

\section{DISCUSSION}

The present study revealed a varied knowledge base (33\% - 83\%) about Tuberculosis (TB) among the study participants. This tells awareness of $\mathrm{TB}$ as an infectious disease among study participants. Literature mentions that the significance of accurate knowledge can influence practices towards the prevention of infectious disease..$^{10}$ The finding of mean knowledge level among participants in the current study (60\%) is better than the study done in Ethiopia $(52 \%)^{2}$ and slightly lower than Iranian (62\%) and Myanmar (72\%) responders from another study. ${ }^{11,12}$ Knowledge about the infectious disease's causal factor is more important as it eventually leads towards preventive practices.

We found that the study participant had a positive attitude (88.1\%) toward dealing with $\mathrm{TB}$ aspects and was aware of $\mathrm{TB}$ as a disease with severe consequences. Among responders, $80 \%$ of participants were aware of free TB treatment at Government facilities, and however, for diagnosed patients, sympathy was expressed by $68 \%$ of the responders. Having diagnosed $\mathrm{TB}$ as an uncomfortable feeling reported in the literature from various studies. ${ }^{2,13,14}$ Despite positive attitudinal values, $23 \%$ of our responders have expressed the possibility of stigma while dealing with patients, which may vary in other studies reported from India. ${ }^{5,7}$

BCG vaccination to children at home were confirmed by $82 \%$ of the responders. Simultaneously, almost all had hygiene maintained $(99.7 \%)$ at their place with $(87.3 \%)$ adequately ventilated house. Since many of the responders were not related to healthcare services, they encountered a health education program of $13.8 \%$. For practice-related aspects, we found that being urban educated and economically stable healthy practices were in place; however, associated stigma with known TB patients was expressed by one in four individuals. An effort to de-stigmatization may be needed, and other studies from the Indian context mention it. Related things are mentioned in national guidelines with TB-related reports. ${ }^{4,5,15,16}$ Health stigma-related aspects need to be evaluated specifically; however, such aspects are beyond this study's scope. We also acknowledge that the study population doesn't involve all Indian populations, and generalizations of findings may need to be considered in a specified context. This study, however, concludes that participants had overall good knowledge (60\%) about Tuberculosis, its causation, prevention, and related factors. In comparison, participants report positive attitudes and related practices. 
Table 1: Socio-economic Distribution of participants characteristics and Knowledge, Attitude and Practices towards TB.

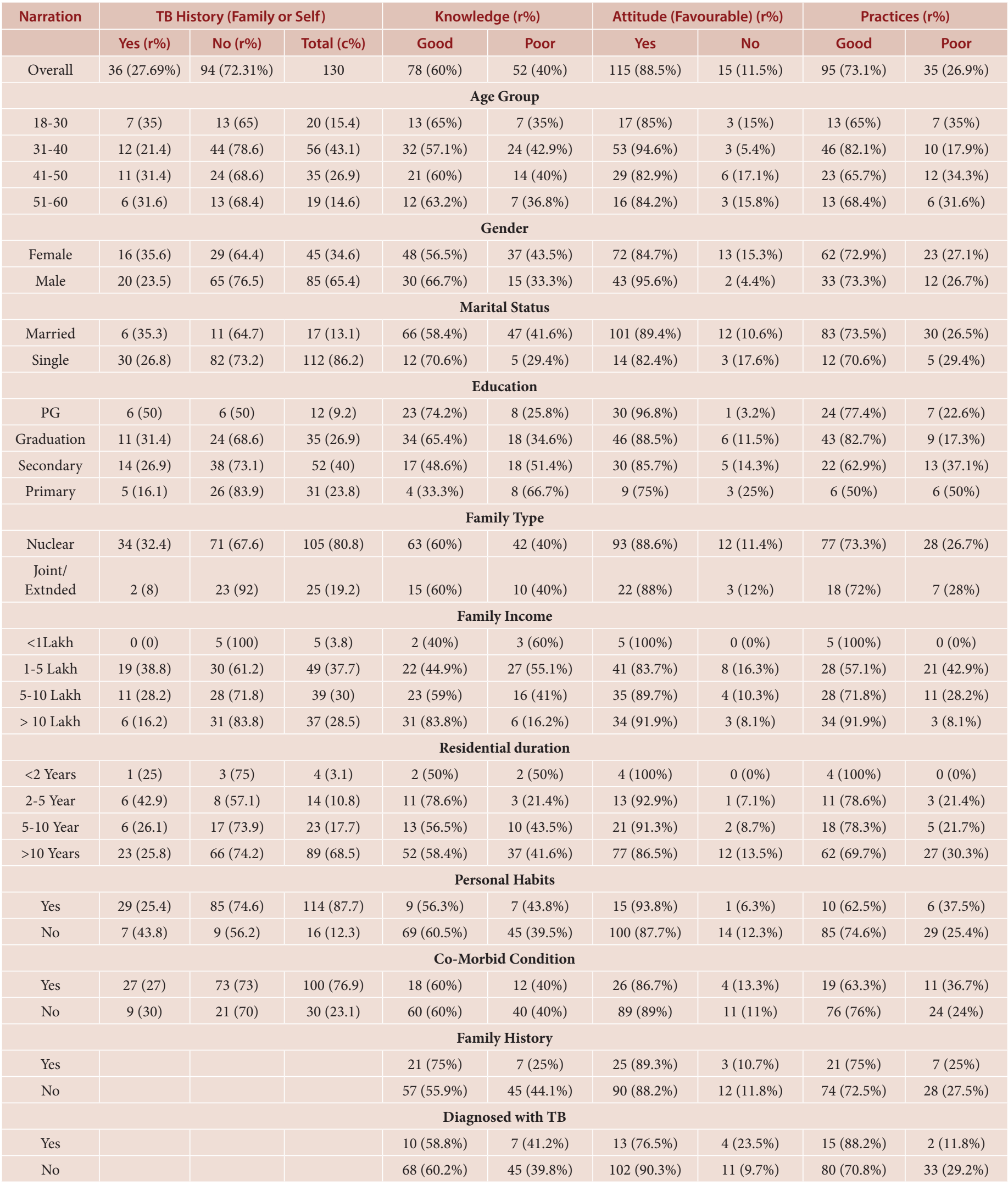

Note: R\%: Row percetages across subgroups, C\%: Column percentage 
Table 2: Questionnaire and respondents with correct responses.

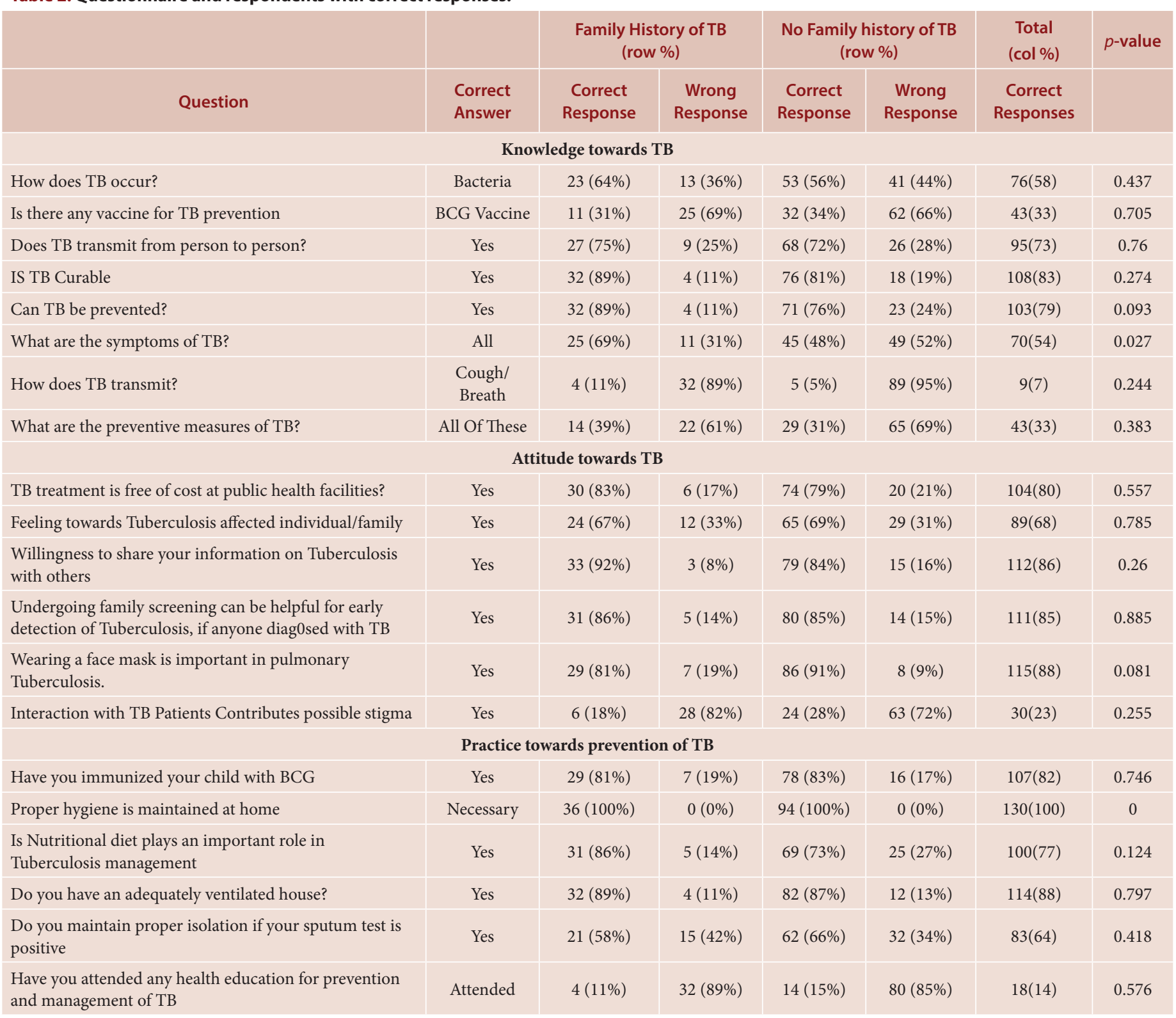

\section{CONCLUSION}

Our findings highlight the scope towards the improvement in the awareness about the disease TB. A positive attitude plays a pivotal role and needs evaluation and consideration masses to lead to good practices in the management of TB patients in the community. Such research emerged as an evaluation step towards the Revised National TB Control Programme (RNTCP) of the national government. Larger evaluation across the rural and urban populations is warranted.

\section{REFERENCES}

1. MoHFW, Central TB Division [internet] [cited Mar 25 2021]. Available from: https://tbcindia.gov.in/.

2. Kasa AS, Minibel A, Bantie GM. Knowledge, attitude and preventive practice towards tuberculosis among clients visiting public health facilities. BMC Res Notes. 2019;12(1):276. doi: 10.1186/s13104-019-4292-2, PMID 31092282.

3. GBD 2019 Diseases and Injuries Collaborators. Global burden of 369 diseases and injuries in 204 countries and territories, 1990-2019: A systematic analysis for the Global Burden of Disease Study 2019. Lancet. 2020;396(10258):1204-22. doi: 10.1016/S0140-6736(20)30925-9, PMID 33069326.

4. Central TB Division. India TB report. Vol. 2018; 2018-. National report [internet]. Available from: https://tbcindia.gov.in/showfile.php?lid=3314 [cited 6/12/2021].

5. G AG, Gopalakrishnan S, Umadevi R. Knowledge, attitude and practices regarding pulmonary tuberculosis in a rural area of Tamil nadu, India: A cross sectional study. 2018;5(9):4055-64.

6. Somma D, Thomas BE, Karim F, Kemp J, Arias N, Auer C, et al. Gender and socio-cultural determinants of TB-related stigma in Bangladesh, India, Malawi and Colombia. Int J Tuberc Lung Dis. 2008;12(7):856-66. PMID 18544216.

7. Sagili KD, Satyanarayana S, Chadha SS. Associated with stigmatising and discriminating attitudes of general population towards tuberculosis patients? Findings from a Community based Survey in 30 Districts of India. 2016;31:1-11.

8. Nadkarni S, Patil P, Roy N, Gerdin M, Bhandarkar P. Eight year longitudinal study of cataract surgeries in an urban Indian population. Int J Community Med Public Health. 2019;6(6, Jun):2019DO - 1018203/2394-6040.ijcmph20192314 [Internet], doi: 10.18203/2394-6040.ijcmph20192314.

9. Bhandarkar P, Gadgil A, Patil P, Mohan M, Roy N. Estimation of the national surgical needs in India by enumerating the surgical procedures in an urban community under universal health coverage. World J Surg. 2021;45(1):33-40. doi: 10.1007/s00268-020-05794-7, PMID 32974741 
10. Launiala A, Honkasalo ML. Ethnographic study of factors influencing compliance to intermittent preventive treatment of malaria during pregnancy among Yao women in rural Malawi. Trans R Soc Trop Med Hyg. 2007;101(10):980-9. doi: 10.1016/j.trstmh.2007.04.005, PMID 17658564.

11. Bagheri Amiri F, Doosti-Irani A, Sedaghat A, Fahimfar N, Mostafavi E. Knowledge, Attitude, and Practices Regarding HIV and TB Among Homeless People in Tehran, Iran. Int J Health Policy Manag. 2018;7(6):549-55. doi: 10.15171/ijhpm.2017.129. PMID 29935132.

12. Yasri S, Wiwanitkit V. Knowledge on tuberculosis in rural Myanmar. Int J Mycobacteriol. 2017;6(4):412-3. doi: 10.4103/ijmy.ijmy_135_17, PMID 29171460.
13. Tolossa D, Medhin G, Legesse M. Community knowledge, attitude, and practices towards tuberculosis in Shinile town, Somali regional state, eastern Ethiopia: A cross-sectional study. BMC Public Health. 2014;14:804. doi: 10.1186/1471-2458-14-804, PMID 25099209.

14. KoayTK. Knowledge and attitudes towards tuberculosis among the people living in Kudat District, Sabah. Med J Malaysia. 2004;59(4):502-11. PMID 15779583.

15. Uplekar M, Weil D, Lonnroth K, Jaramillo E, Lienhardt C, Dias HM, et al. Viewpoint WHO's new End TB Strategy; 2015;1799-801.

16. Malhotra R, Taneja OK, Dhingra VK, Rajpal S, Mehra M. Awareness regarding tuberculosis in A rural population of Delhi. Indian J Commun Med. 2002;27(2):62

Cite this article : Laiby R, Debjani P, Prashant B. Knowledge, Attitude and Practices Towards Tuberculosis: Study Amongst Urban Adults Visiting the Community Health Center. Int J Med Public Health. 2022;12(1):28-32. 